\title{
Probing the gluon plasma with charm balance functions
}

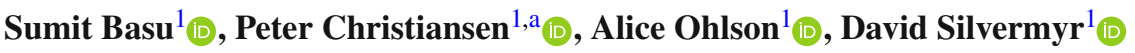 \\ ${ }^{1}$ Division of Particle Physics, Department of Physics, Lund University, Box 118, 22100 Lund, Sweden
}

Received: 22 June 2021 / Accepted: 5 November 2021 / Published online: 23 November 2021

(C) The Author(s) 2021

\begin{abstract}
Recent theoretical explanations for how hydrodynamic-like flow can build up quickly in small collision systems (hydrodynamization) has led to a microscopic picture of flow building up in a gluon-dominated phase before chemical equilibrium between quarks and gluons has been attained. The goal of this contribution to Offshell-2021 is to explore consequence of assuming a long-lived gluondominated phase, which we shall denote a gluon plasma (GP). As these consequences are naturally enhanced in a large systems, we assume and explore the extreme scenario in which a GP would be created in AA collisions and exist for significant time before the formation of a chemicallyequilibrated quark-gluon plasma (QGP). The GP and its formation would be impossible to probe with light-quark hadrons, which are first produced later in this scenario. As charm quarks are produced early in the collision, they can circumvent the limitations of light quarks and we propose charm balance functions as an effective tool to test this idea and constrain the dynamics of the GP.
\end{abstract}

\section{Introduction}

One of the biggest open questions in the study of heavy-ion collisions is how the initial interactions lead to the formation of a strongly-interacting medium. When the perfect liquid was discovered at RHIC [1-3] it was conjectured that hydrodynamic behavior implied the formation of a thermal medium. With the discovery of flow in small collision systems [4-6], such as pp and p-Pb collisions, at the LHC, the question of thermalization has become a hot topic since it is hard to understand how a small system can have time to thermalize. It appears that the concept of hydrodynamization must be part of the solution to this open issue [7]. Hydrodynamization is the phenomena that a system out of equilibrium can sometimes still be described by hydrodynamics.

\footnotetext{
a e-mail: peter.christiansen@ @ep.lu.se (corresponding author)
}

Recently, a microscopic picture of hydrodynamization has been developed based on kinetic theory, see e.g. Ref. [8]. In these calculations, the initial state is completely dominated by gluons and it is in this state that the system hydronamizes. Light quarks are eventually produced and the system then first attains chemical and then later thermal equilibration (see Ref. [8] for a detailed discussion). This picture is very similar to the 30-year-old idea of "Hot Glue" [9] and more recent versions like "Undersaturated QGP" [10], which are all driven by the expectation that the initial state will be gluon-dominated due to the fact that the magnitude of the color charge of the gluons is approximately twice that of the quarks. As a consequence, the initial projectile/target wave functions become gluon dominated at large beam energies and at the same time gluons are more likely to scatter or be produced in an annihilation process both in initial and final state interactions. While there has been some previous work on this idea, we stress that this is not the standard picture of QGP formation.

In this paper, we resurrect these speculative ideas and reexamine them. We think it is timely to do so because of the recent realization that hydrodynamic flow does not require thermalization. This realization means that the system can exhibit properties traditionally associated with a QGP medium - namely, collective flow (in kinetic equilibrium) but not yet be in chemical or thermal equilibrium, which is unlike the standard QGP picture of the last 20 years. If such a system is indeed produced, and it has QGP-like properties but is gluon-dominated, we propose to denote it the gluon plasma (GP) and reserve QGP for the chemically-equilibrated system. The main goal of this paper is to point out that the possible existence of a GP phase leads to a different view of classic QGP observations and to identify how we can get further insights into this idea experimentally.

Before we go on, we stress that in a weakly-coupled calculation (e.g. as discussed in Ref. [8]) one would not expect a significant impact of a GP/"Hot Glue" phase. However, the weak coupling assumption is questionable [11] and likely 
enforced by the lack of theoretical tools to do the stronglycoupled microscopic calculation. We therefore here assume and explore a more extreme strongly-coupled scenario where the GP phase would last for a significant time and dominate the initial system. In this way, we can point out both the strengths of such a hypothetical picture and how we can test it experimentally.

As we shall see, the existence of a significant GP phase could provide novel insights into some open theoretical issues, but also presents experimental challenges: if true, it would imply that light quarks are produced late in the collision evolution and so light hadrons will likely be less sensitive to early-time dynamics. For this reason, we propose to use low $p_{\mathrm{T}}$ charm quarks to study the dynamics of the hydrodynamization process. The advantage of using charm quarks is that:

- they are dominantly produced early in the collision with rates that are calculable in perturbative QCD (pQCD).

- they are the most abundantly produced heavy quarks.

- they are known to interact with the medium and exhibit collective flow [12].

For completeness, we note that the charm quantum number, $C$, is conserved by strong and electromagnetic interactions while it can be violated in weak decays. This means that charm quarks are essentially always created in pairs $(c \bar{c})$. As weak decays predominantly occur over longer timescales than the hadronization process, it is still possible to reconstruct the original charm hadrons and determine their charm content $(c \text { or } \bar{c})^{1}$.

This paper is organized as follows: Sect. 2 describes the difference between the GP and the QGP and discusses general concepts used in the rest of the paper. Section 3 focuses on the idea to study the charm-anticharm balance functions. In Sect. 4 we discuss correlations between charm hadrons and "regenerated" $J / \psi$ and finally in Sect. 5 we summarize the main conclusions of the paper.

\section{The gluon plasma vs the quark-gluon plasma}

In this section we discuss the idea that heavy-ion collisions lead to the early formation of a GP and it is only later, after significant flow has been built up in the system, that a QGP is formed. The goal is to try to convince the reader that there are some existing results that can be more easily understood if the initial system formed is a GP.

The essential differences that a GP introduces, with respect to the standard QGP picture, are:

\footnotetext{
$\overline{{ }^{1} \text { For neutral } D}$ mesons, one can have oscillations, e.g., $D^{0}-\bar{D}^{0}$ oscillations, which violate $C$, but this effect is essentially negligible.
}

- an absence of light quarks at early times when the system is the hottest.

- that light quarks are created flowing.

Two heavy-ion observables that are the most sensitive to early light quark dynamics are thermal photon production and the chiral magnetic effect.

Thermal photons ${ }^{2}$ have been used to study the thermal properties of the initial state $[13,14]$. It is clear that photons have no direct coupling to gluons, which are electrically neutral, and so one would naively expect that the dominant source of thermal photons will be thermal radiation from light quarks. If a GP precedes the QGP phase, then one would expect thermal photons to have a lower temperature because they would only be produced later in the evolution of the system after the "fireball" has already expanded and cooled, and that they will be produced from a flowing system and thus exhibit collective flow behavior. While it is unclear if the measured photon spectrum is cooler than expected, it was a huge surprise that the thermal photons exhibited azimuthal anisotropic flow in heavy-ion collisions [15]. This puzzle is still unresolved and the existence of a GP phase may be a key to resolve this question. These ideas have already been explored in a similar context in Refs. [16,17].

The observation of the chiral magnetic effect (CME) would be a signature of parity violation in the strong interaction. The CME is postulated to arise if there are topological regions within the QGP with non-zero net chirality (i.e. an excess or depletion of left- or right-handed quarks). Such domains may result in an experimentally-observable asymmetry in charged particle emission because they would exist in the presence of a huge magnetic field which arises at very early times from the angular momentum of the noninteracting spectators in non-central heavy-ion collisions. The electric dipole, which would be created by light quarks aligning their spins with this magnetic field, would result in a charge splitting in the direction of the magnetic field. For a review, see Refs. [18,19].

While the CME has been observed in semimetals (e.g. $\mathrm{ZrTe}_{5}$ [20]), the experimental search for such charge splitting in heavy-ion collisions has been challenging due to the presence of background effects like collective flow and local charge conservation which can mimic CME-like signals. Recent efforts to measure the CME and estimate the level of background contributions indicate that at most only a small fraction of the observed CME-like signals may be attributable to chiral effects [21-23]. This lack of a significant observed CME signal $[21,24]$ may also be consistent

\footnotetext{
${ }^{2}$ Experimentally, one measures direct (non-decay) photons, which are expected to be a mix of prompt photons (dominant at high $p_{\mathrm{T}}$ ) and thermal photons (dominant at low $p_{\mathrm{T}}$ in central AA collisions).
} 


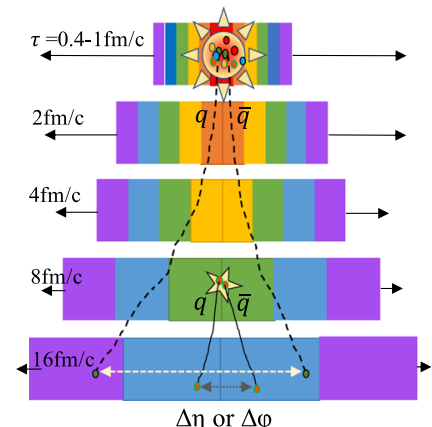

(a) Clocking Hadronization of $q \bar{q}$ pairs

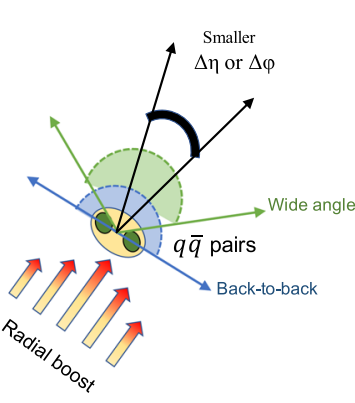

(b) Kinematic Lensing due to radial boost
Fig. 1 Illustration of the physics affecting the width of the balance function. a Charges produced early in the collision will be further separated in momentum space than charges produced later (Inspired by Ref. [25]). b Radial flow can lead to a narrowing of the balance function due to kinematic lensing or focusing (Inspired by Fig. 9 in Ref. [26])

with the picture that the early stages of the heavy-ion collision system are a GP phase, where quarks have not yet been created. We are not aware of anyone else pointing out this possibility but we think the GP solution to the CME puzzle is very much in the spirit of the rest of this paper because it is not that there is no CME, it is rather that there are no quarks to probe it!

Another result that is very important for the discussion in the next section is related to balance functions [25,27-29]. Balance functions are used to measure how far in momentumspace one has to go to balance quantum numbers, e.g., the charge balance function measures where in momentumspace the electric charge is balanced. Figure 1 gives an illustration of what physics affects the width of the charge balance functions:

- Clocking Hadronization: the width is broader (narrower) if the quarks that make up the hadrons are produced early (late), as they have more (less) time to diffuse [25].

- Kinematic Lensing: the angle between pairs of particles created at rest in the comoving frame will be narrowed (focused) when boosted to the lab frame [30].

The top and bottom panels of Fig. 2 present experimental results for the charge balance function in $\sqrt{s_{\mathrm{NN}}}=2.76 \mathrm{TeV}$ $\mathrm{Pb}-\mathrm{Pb}$ collisions. It is observed that for more central, longerlived collisions, the balance function is narrower. In the QGP picture, the interpretation is that, even if quarks are produced early, they stay in thermal equilibrium (pairs are created and annihilated) until hadronization and so the charge balance is produced late when the system is flowing. In this picture, the balance would be produced at a similar $T_{c}$ for all centralities (late clocking hadronization) and the main difference is then due to the different amount of Kinematic Lensing. In the

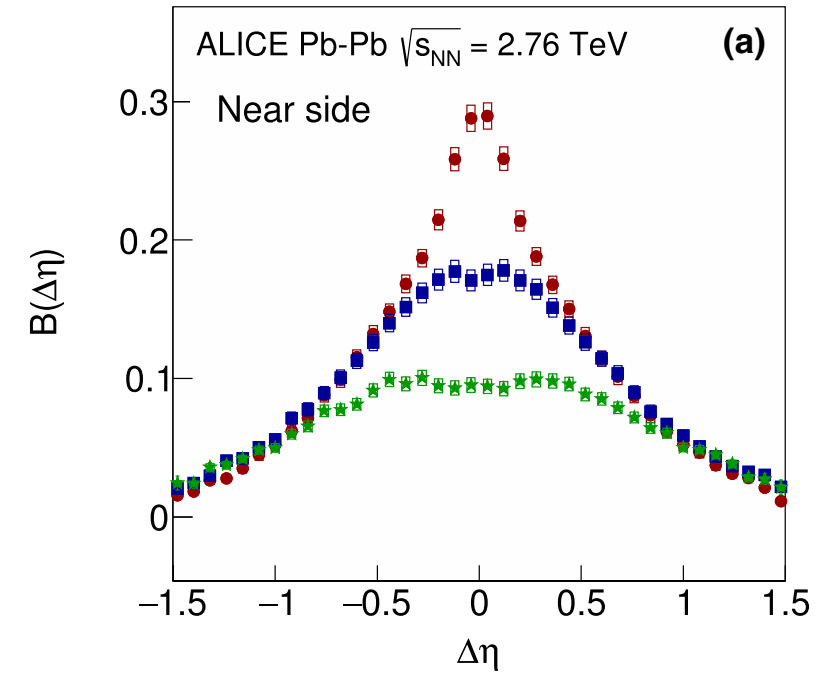

ALI-PUB-103502

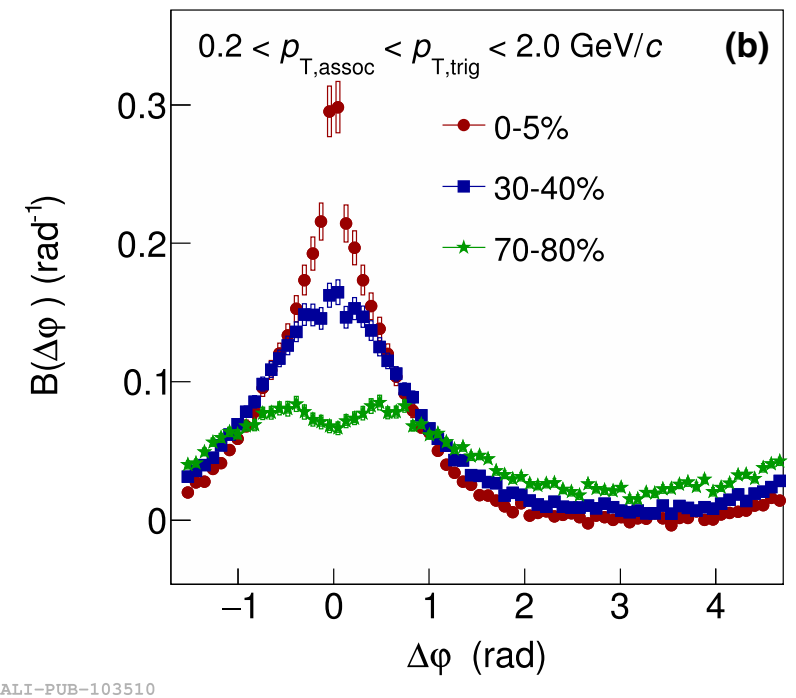

Fig. 2 Top: the balance functions in $\sqrt{s_{\mathrm{NN}}}=2.76 \mathrm{TeV} \mathrm{Pb}-\mathrm{Pb}$ collisions as a function of the pseudorapidity separation between two charged particles on the near side (for relative azimuthal separations $-\pi / 2<\Delta \varphi<\pi / 2$ ). One observes a clear narrowing in central collisions. Taken from Ref. [31]. Bottom: similar to the top plot but for separation in azimuthal angle

GP picture the balance of light quarks is naturally created late when the system is already flowing and so one would reach a similar conclusion but without the requirement that quarks are balanced down to $T_{c}$, which seems to be in contrast with the widespread idea of a grand canonical ensemble. It might seem that the narrowing of the charge balance function is of little importance but one of the main signatures of a QGP must be deconfinement, i.e. that pairs of quarks created together at early times will hadronize far apart at later times. This type of deconfinement is exactly what differentiates the QGP from confined descriptions, such as the Lund string model [32-34]. The irony of the GP scenario would be that the initial state is made up of deconfined gluons but that 
we cannot easily measure this deconfinement, since we are predominantly sensitive to the quarks (hadrons), which are produced late and kinematically focused. In Sect. 3 we therefore extend the balance function to charm-anticharm quarks which are produced early in the collision and therefore should behave completely differently from the light hadrons shown in Fig. 2.

Finally we touch upon two ideas that we hope can inspire the reader.

1. If a GP phase exists, it will not be described by standard lattice QCD (LQCD) calculations as it is out of chemical equilibrium and it could imply that the screening of the $c \bar{c}$ potential is not well described by LQCD.

2. The focus in the following will be dominantly on LHC physics. However, we note that there could be a difference between the relative importance of the GP at the LHC, RHIC, and the SPS, as one would expect the initial states to be more quark-dominated at lower beam energies.

\section{Charm-anticharm balance functions}

Heavy quarks have played an important role in studies of the QGP for a long time. Initially, quarkonia were mainly studied due to the predicted and observed melting in a deconfined medium [35-37]. However, in particular at LHC energies, the charm mesons have become a hot topic because they are welldefined probes of the produced medium: the initial charm quark production can be precisely estimated in heavy-ion collisions, their mass is large with respect to the temperature of the produced medium and a large fraction of the charm quarks are non-relativistic. For a recent overview, see for example Ref. [38].

So far, experimental measurements have primarily focused on the spectra of charm hadrons, but these provide only coarse information about final state interactions and one must now go on to study correlations. In the GP picture, correlating charm hadrons with light-flavor hadrons (as has been done in both correlation and flow measurements) does not probe the build-up of flow signals because the light quarks are produced after the system is already flowing. Therefore we think that in order to access the earliest stages of the collision, one must now go on to study correlations between charm hadrons.

For charm-(anti)charm correlations, we first point out that one can in principle calculate the initial momentum correlations between $c \bar{c}$ pairs using pQCD, see e.g. Ref. [39]. The initial momentum correlations will be the same in peripheral and central heavy-ion collisions because the microscopic scattering process is the same, and charm quarks from independent scatterings will be uncorrelated in momentum space. The production rate will be scaled up to account for the nuclear overlap geometry. We also mention that phenomeno- logical studies of charm-charm correlation functions have previously been done for flow [40], thermalization [41,42], jet quenching [26], and even for the effects of pre-equilibrium physics [43]. The idea to study charm-charm correlation functions (and even balance functions) is therefore not new and even the idea to use it as a probe of early-time physics can be found in some of these papers. What is new in this paper is the idea of the GP and how to probe it with charm balance functions, the specific ideas of Sect. 4, but maybe most importantly we hope to convince the reader that these types of correlation measurements, that are extremely challenging from an experimental point of view, are no longer "nice to have" but really something we "need to have" as a community.

\subsection{What type of correlations should be measured?}

We propose to measure the correlation between the charm and the anticharm quarks produced in the same hard process. It is an extension of ideas described in Ref. [44] for strange-antistrange correlations and we propose to use the same analysis method as described in Refs. [45,46].

First of all, one cannot measure the charm quarks directly and so one has to measure the charm hadrons, e.g., $D^{0}(c \bar{u})$ and $\bar{D}^{0}(\bar{c} u)$. In the model calculations shown in this paper we will generally just sum over all charm hadrons and all anticharm hadrons for the results but this of course cannot be done experimentally. Secondly, one does not know if a given charm hadron and a particular anticharm hadron are from the same initial process. To access the directly-correlated pairs we propose to do as in Refs. [45,46] and subtract charmcharm and anticharm-anticharm correlations from charmanticharm correlations. In this way, and if we properly normalize the correlation functions, we are left with the direct correlations, which is the balance function as described in Sect. 2. We will therefore be able to access the early-time dynamics (see Fig. 2, top panel) by measuring how the balance function in heavy-ion collisions is modified with the respect to the one calculable (measurable) in pQCD (pp collisions). We note that there are slightly different ways to define and normalize the balance function mathematically, see for example Refs. [25,30,31,47], but that they are in essence the same and boil down to measuring experimentally where in momentum-space the charm quantum number is balanced.

To illustrate the idea behind the charm balance function, in particular the subtraction scheme, we have carried out a simulation study using PYTHIA $[34,48]$. We have generated events where we have forced the hardest scattering to produce a pair of $c \bar{c}$ quarks. In some cases more than one pair of charm hadrons have been produced $(c \bar{c}$ can also be produced in sub-leading hard processes). These events have been rejected to ensure that we always have only one pair 

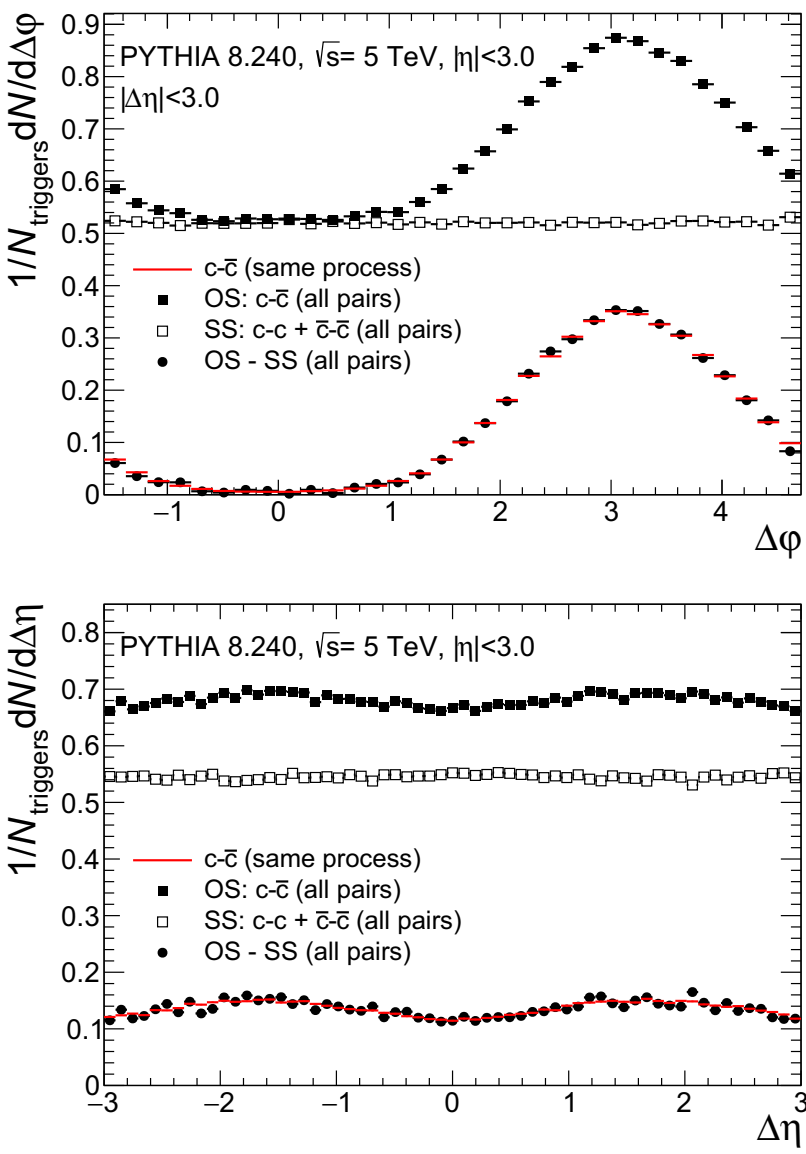

Fig. 3 Illustration of how one experimentally can extract the correlation between correlated charm and anticharm quarks produced in the same hard process (red line). The experimental observable (OS-SS) is constructed by subtracting the same sign (SS) per trigger yield from the opposite sign (OS) per trigger yield. Top: azimuthal correlations. Bottom: pseudorapidity correlations. Note that results are shown for all charm hadrons and that $c$ and $\bar{c}$ only refers to the charm content of the hadrons

of correlated charm quarks, but we note that for these events there was no angular correlation between the leading and sub-leading pairs of charm hadrons. Figure 3 shows the correlation between the charm and anticharm hadrons produced in the same hard scattering (red line). Here we point out that in Fig. 3, the charm and anticharm hadrons are obviously backto-back in azimuthal angle, but LHCb has actually measured these correlations in pp collisions and found a significant near-side peak [49]. To get such a near-side peak one would need significant NLO corrections as discussed in Ref. [39]. So one should be aware that the real correlations could be very different from the ones obtained at LO in PYTHIA. In fact, one could wonder if it would be possible to experimentally constrain NLO contributions to the charm production process using this type of correlation measurement. We note that since the correlations in pp collisions are mainly consid- ered here as a reference for AA measurements, this has no impact on the arguments that follow.

One challenge in obtaining the correlation function shown in Fig. 3 is that we will experimentally measure both the correlated and uncorrelated pairs. Here we show our idea for how to remove the uncorrelated pairs experimentally: To construct the correlation function, we mix each event with 6 other random events and measure that same sign (SS) and opposite sign (OS) per-trigger yields. Now, by subtracting the SS from the OS yields one can in fact recover the directly correlated yield between the charm and the anticharm quarks, which we will call the charm-anticharm balance function in the following.

We have not included detailed model predictions for what one will be able to measure using the charm-anticharm balance function. The concept of the GP is a novel idea and it is not yet implemented in any standard generators, but we expect that one should be able to get some idea about this in kinetic theory, e.g., using the formalism of Ref. [8]. We also note that an out-of-equilibrium calculation, using the Glasma formalism, was presented in Ref. [43]. At the qualitative level, we predict that one should observe something very different from what was measured for light quarks, cf. Fig 2. In fact, in simulations done for jet quenching, which is modeled as an interaction between the medium and the pair of charm quarks in Ref. [26], then a broadening of the balance function is observed. We think that the observation of a broadening at low $p_{\mathrm{T}}$ would establish that the physics one can extract from the charm-anticharm balance function would be of a different nature from what the light quarks probe.

Finally, we reiterate the main ideas here:

- Charm is dominantly produced in hard scatterings in the initial stages of the collision, which means that one can establish a precise charm balance function reference in small systems.

- It is known that charm interacts with the medium as it builds up flow.

- We propose to measure how the charm balance function is modified as the system size is increased.

Depending on what kind of statistics can be achieved, one could even imagine combining this with event-shape engineering [50] to ensure that all initial states have similar geometries while one mainly varies the system lifetime.

\section{Correlations between $J / \psi$ and charmed hadrons}

The production of charmonium in general, and $J / \psi$ in particular, is one of the most studied signatures in heavy-ion collisions, since the production is expected to be affected 


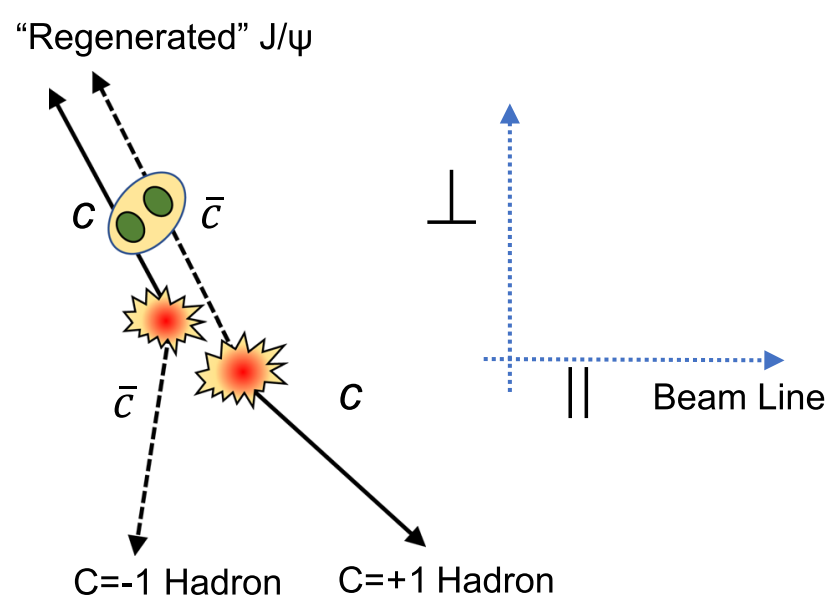

Fig. 4 Illustration of the $J / \psi$ "regeneration" and how it leads to correlations between the $J / \psi$ and the "associated" charm hadrons

by color screening in the dense QGP state [35]. At the LHC, it is observed that the $J / \psi$ nuclear modification factor $R_{\mathrm{AA}}{ }^{3}$ is larger than what has been measured at RHIC at low $p_{\mathrm{T}}[51,52]$. One normally expects that at higher beam energies, the plasma is hotter and therefore the screening/suppression should be more significant. To explain this difference it has been proposed that $J / \psi$ "regeneration" plays a large role at LHC: if charm is copiously produced it can occur that a charm quark produced in one hard scattering can combine with an anticharm quark produced in another independent hard scattering to form a $J / \psi$. Since charm is more abundantly produced at LHC energies than at RHIC, the larger effect of regeneration, on top of the expected suppression, leads to a higher $R_{\mathrm{AA}}$ at the LHC [53].

If $J / \psi$ regenerates at the LHC, it would be a direct signature that charm/anticharm quarks are deconfined in the plasma. Therefore one should consider if there are alternative explanations. A challenge in interpreting $R_{\mathrm{AA}}$ measurements is that it depends on the beam energy, since the pp reference spectrum will typically harden as the beam energy increases. The same modification of the AA spectrum at two different beam energies can therefore lead to two different values of $R_{\mathrm{AA}}$. An alternative reason for the change in the $J / \psi R_{\mathrm{AA}}$ from RHIC to the LHC could be if GP effects play a larger role at the LHC and result in less screening of the charmonium potential. For this reason, we propose in the following to search for correlations between $J / \psi$ from regeneration and other charm hadrons.

Figure 4 illustrates how $J / \psi$ regeneration leads to correlations between the $J / \psi$ and charm hadrons. What is important to stress here is that one normally does not expect such cor-

\footnotetext{
${ }^{3} R_{\mathrm{AA}}$ quantifies the modification of hard processes in AA collisions with respect to an independent superposition of pp collisions, taking into account the enhanced production due to the nuclear geometry; see e.g. Ref. [38] for details.
}
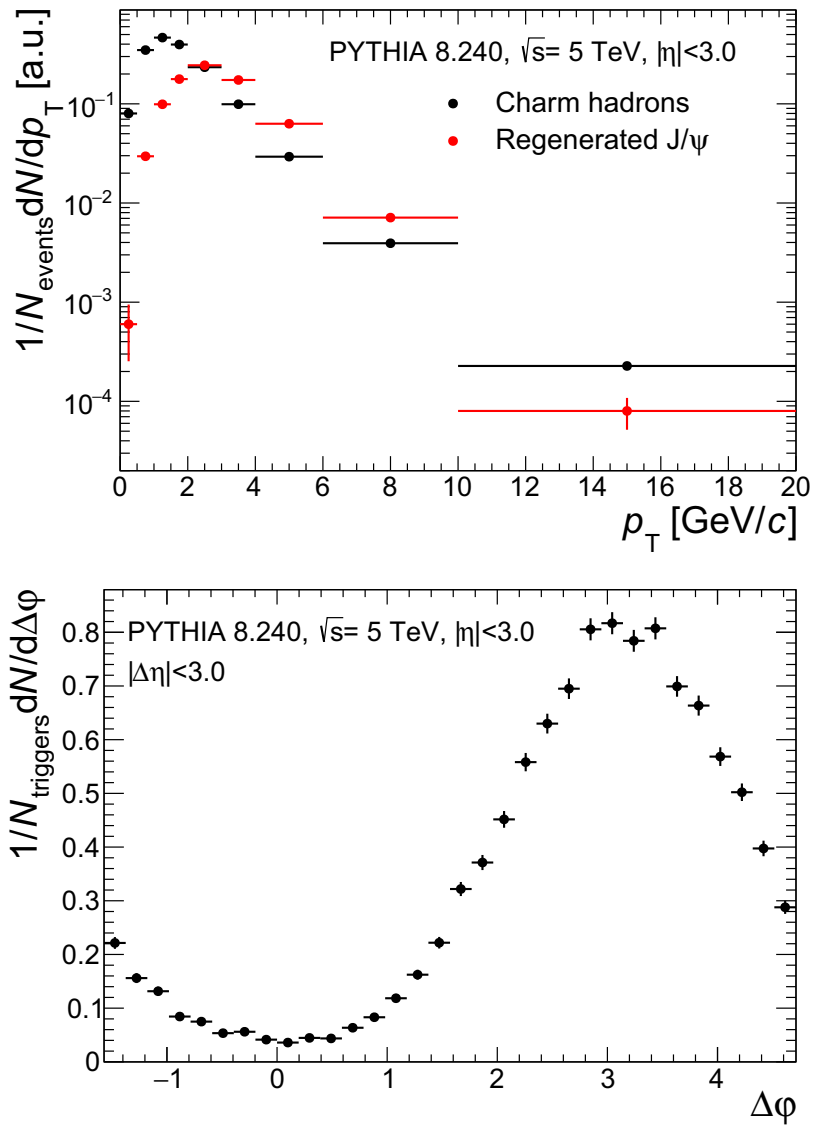

Fig. 5 Predictions for regenerated $J / \psi$ hadrons based on PYTHIA simulations, see text for details. Top: the comparison between the $p_{\mathrm{T}}$ spectra for charmed "quarks" and regenerated $J / \psi$. Note that the spectra have been scaled to have a similar magnitude so the shape is easy to compare. Bottom: azimuthal correlations between regenerated $J / \psi$ and their two correlated charm-hadron partners

relations to be present as the $J / \psi$ is expected to be directly produced. LHCb has in fact measured correlations between $J / \psi$ and D mesons and this correlation is approximately flat in azimuthal angle [49], indicating that there are little or no direct correlations between these hadrons under normal circumstances. We therefore propose to study $J / \psi$-charmhadron correlations to validate that indeed $J / \psi$ regeneration is the explanation for the increase in $R_{\mathrm{AA}}$ at low $p_{\mathrm{T}}$.

To estimate how these correlations could look, we have utilized the PYTHIA simulations discussed in the previous section. We have matched charm "quarks" 4 from two different pp (nucleon-nucleon) collisions by requiring that their rapidity, azimuthal angle and $p_{\mathrm{T}}$ are close. In the case that the two "quarks" are close we have combined them into a $J / \psi$ with similar angle and rapidity and approximately twice the $p_{\mathrm{T}}$. Then we have measured the angular correlations with respect to the remaining "associated" charm hadrons in the event. Figure 5 shows the $p_{\mathrm{T}}$ spectrum of the regenerated

\footnotetext{
${ }^{4}$ We used charm hadrons as proxies for the quarks.
} 
$J / \psi$ (top) and the azimuthal correlation between the $J / \psi$ and the "associated" charm hadrons (bottom). As can be seen, there is a strong correlation on the away side. The large magnitude is easy to understand, as each regenerated $J / \psi$ will have two charm hadron partners, and that it is primarily on the away side is easy to understand from Fig. 5. Due to possible NLO corrections discussed previously, an experimentally observed balance will not necessarily be on the awayside peak only. However, we note that the expected shape is in principle derivable from the measured charm spectra and charm-anticharm balance function, discussed in Sect. 3. We therefore think that these measurements can provide clear and unique signatures of $J / \psi$ regeneration. The only exception to this would be if charm quarks fully kinematically (but not chemically) thermalize as discussed in Ref. [53]. But again, this would be observable in measurements of the charm balance function, which in this case would have no structure. We note that we consider such a scenario very unlikely as what makes the GP/QGP liquid perfect is exactly that the effect of dissipation and diffusion is as low as possible.

In this paragraph we will go into detail about how we think one could search for this signature in Runs 3 and 4 at the LHC. To search for the largest signal, one will have to study central collisions where regeneration is the largest effect and trigger on low $p_{\mathrm{T}} J / \psi$ where one knows that the regeneration plays a big role and where the $c \bar{c}$ pairs are produced backto-back. An important crosscheck that a signal is indeed real and from regeneration will be that one can switch it off both by going to peripheral collisions and by increasing the trigger $p_{\mathrm{T}}$. The final thing to consider is what hadrons to measure. The correlation function in Fig. 5 (bottom) is for all charm hadron species. The important thing will be to try recover as much as possible of the yield of charm hadrons.

In the final part of this section we will go through how we think these measurements can provide alternative insights. First, we note that the measurements described here have a strong relation to the balance function measurements described in Sect. 3: in this section we measure how the regenerated $J / \psi$ is balanced by its original charm partners. Therefore, the results should be able to provide similar information as discussed in the previous section. Furthermore, we think that if one would establish this as a unique signature of $J / \psi$ regeneration then one could even use this signature to search for similar effects in small collision systems. In this way one could hope to find evidence of deconfinement even in small systems. We note that there are ideas similar to $J / \psi$ regeneration even in PYTHIA where it can occur via a process known as "cluster collapse" [54]. Cluster collapse can occur when "the string mass is so small that the cluster cannot decay into two hadrons. It is then assumed to collapse directly into a hadron resonance, inheriting the flavor content of the string endpoints", (see Ref. [54] for details). This normally does not produce $J / \psi$ as the charm and anticharm pro- duced in a single scattering will end up on different strings. However, in case two or more $c \bar{c}$ pairs are produced, $J / \psi$ production can occur due to color reconnection as discussed in detail in Ref. [55]. We would expect this type of $J / \psi$ production mechanism to have similar signatures as shown in Figs. 4 and 5 and note that this signature, as far as we know, is discussed for the first time here.

One could argue that the PYTHIA implementation raises questions about the unique interpretation of $J / \psi$ regeneration as a signature of deconfinement. It should be clear that partons in the GP and the QGP are strongly interacting so deconfinement does not mean "free". We think the main point here is that in AA collisions the magnitude of the $J / \psi$ regeneration requires that $c \bar{c}$ pairs from different nucleon-nucleon collisions are required to "regenerate", which to us means that the charm quarks are not confined together with the partons produced locally but can end up in hadrons with quarks produced in processes that are, at least in essence, causally separated. For a similar signature in a small system one would not be able to use this argument and would instead have to determine what is the most likely scenario: to have one or two production mechanisms.

\section{Conclusions}

In this paper, we have drawn attention to the possible existence of a gluonic state, which we have denoted the gluon plasma (GP), that could dominate early-time dynamics of hadronic collisions. The dynamics of the GP, which is critical for understanding the build up of flow, might therefore be invisible to light quark hadrons as they are first produced after the system is already flowing. We have argued that the evolution of the charm balance function as one goes from small to large systems would be sensitive to the early time GP dynamics and therefore charm-charm correlation functions will be vital measurements in the LHC physics program during Runs 3 and 4.

The question of the GP is fundamentally linked to the question of deconfinement as the GP will effectively give similar experimental signatures as confined models. For this reason, we have in the latter part shown exactly how one can combine the ideas of the charm balance function with $J / \psi$ regeneration to strengthen the experimental observation of charm quark deconfinement.

Acknowledgements $\mathrm{PC}$ and $\mathrm{AO}$ gratefully acknowledge funding from the Knut and Alice Wallenberg Foundation (the CLASH project).

Data Availability Statement This manuscript has no associated data or the data will not be deposited. [Authors' comment: We do not have any extra data to display. The data used in the manuscript are taken from published publicly available data from ALICE \& LHCb collaborations and sources are cited in bibliography.] 
Open Access This article is licensed under a Creative Commons Attribution 4.0 International License, which permits use, sharing, adaptation, distribution and reproduction in any medium or format, as long as you give appropriate credit to the original author(s) and the source, provide a link to the Creative Commons licence, and indicate if changes were made. The images or other third party material in this article are included in the article's Creative Commons licence, unless indicated otherwise in a credit line to the material. If material is not included in the article's Creative Commons licence and your intended use is not permitted by statutory regulation or exceeds the permitted use, you will need to obtain permission directly from the copyright holder. To view a copy of this licence, visit http://creativecomm ons.org/licenses/by/4.0/.

Funded by SCOAP ${ }^{3}$.

\section{References}

1. J. Adams et al., Nucl. Phys. A 757, 102 (2005). https://doi.org/10. 1016/j.nuclphysa.2005.03.085

2. K. Adcox et al., Nucl. Phys. A 757, 184 (2005). https://doi.org/10. 1016/j.nuclphysa.2005.03.086

3. W. Busza, K. Rajagopal, W. van der Schee, Ann. Rev. Nucl. Part. Sci. 68, 339 (2018). https://doi.org/10.1146/ annurev-nucl-101917-020852

4. V. Khachatryan et al., JHEP 09, 091 (2010). https://doi.org/10. 1007/JHEP09(2010)091

5. B. Abelev et al., Phys. Lett. B 719, 29 (2013). https://doi.org/10. 1016/j.physletb.2013.01.012

6. J.L. Nagle, W.A. Zajc, Ann. Rev. Nucl. Part. Sci. 68, 211 (2018), https://doi.org/10.1146/annurev-nucl-101916-123209

7. P. Romatschke, U. Romatschke, Relativistic Fluid Dynamics In and Out of Equilibrium. Cambridge Monographs on Mathematical Physics (Cambridge University Press, 2019). https://doi.org/ 10.1017/9781108651998

8. A. Kurkela, A. Mazeliauskas, Phys. Rev. Lett. 122, 142301 (2019). https://doi.org/10.1103/PhysRevLett.122.142301

9. E.V. Shuryak, Phys. Rev. Lett. 68, 3270 (1992). https://doi.org/10. 1103/PhysRevLett.68.3270

10. H. Stocker et al., Astron. Nachr. 336(8/9), 744 (2015). https://doi. org/10.1002/asna.201512252

11. E. Shuryak, (2019). arXiv:1901.00178 [nucl-th]

12. S. Acharya et al., JHEP 02, 012 (2019). https://doi.org/10.1007/ JHEP02(2019)012

13. A. Adare et al., Phys. Rev. C 81, 034911 (2010). https://doi.org/ 10.1103/PhysRevC.81.034911

14. J. Adam et al., Phys. Lett. B 754, 235 (2016). https://doi.org/10. 1016/j.physletb.2016.01.020

15. A. Adare et al., Phys. Rev. Lett. 109, 122302 (2012). https://doi. org/10.1103/PhysRevLett.109.122302

16. F.M. Liu, S.X. Liu, Phys. Rev. C 89(3), 034906 (2014). https://doi. org/10.1103/PhysRevC.89.034906

17. A. Monnai, Phys. Rev. C 90(2), 021901 (2014). https://doi.org/10. 1103/PhysRevC.90.021901

18. D.E. Kharzeev, Prog. Part. Nucl. Phys. 75, 133 (2014). https://doi. org/10.1016/j.ppnp.2014.01.002

19. D.E. Kharzeev, Ann. Rev. Nucl. Part. Sci. 65, 193 (2015). https:// doi.org/10.1146/annurev-nucl-102313-025420

20. Q. Li, D.E. Kharzeev, C. Zhang, Y. Huang, I. Pletikosic, A.V. Fedorov, R.D. Zhong, J.A. Schneeloch, G.D. Gu, T. Valla, Nat. Phys. 12, 550 (2016). https://doi.org/10.1038/nphys 3648

21. S. Acharya et al., Phys. Lett. B 777, 151 (2018). https://doi.org/10. 1016/j.physletb.2017.12.021

22. V. Khachatryan et al., Phys. Rev. Lett. 118(12), 122301 (2017). https://doi.org/10.1103/PhysRevLett.118.122301
23. S. Acharya et al., JHEP 09, 160 (2020). https://doi.org/10.1007/ JHEP09(2020) 160

24. M. Abdallah et al., (2021). arXiv:2109.00131 [nucl-ex]

25. S.A. Bass, P. Danielewicz, S. Pratt, Phys. Rev. Lett. 85, 2689 (2000). https://doi.org/10.1103/PhysRevLett.85.2689

26. M. Nahrgang, J. Aichelin, P.B. Gossiaux, K. Werner, Phys. Rev. C 90(2), 024907 (2014). https://doi.org/10.1103/PhysRevC.90. 024907

27. S. Jeon, S. Pratt, Phys. Rev. C 65, 044902 (2002). https://doi.org/ 10.1103/PhysRevC.65.044902

28. S. Pratt, W.P. McCormack, C. Ratti, Phys. Rev. C 92, 064905 (2015). https://doi.org/10.1103/PhysRevC.92.064905

29. A. Bialas, Phys. Lett. B 579(1-2), 31 (2004). https://doi.org/10. 1016/j.physletb.2003.10.106

30. B. Abelev et al., Phys. Lett. B 723, 267 (2013). https://doi.org/10. 1016/j.physletb.2013.05.039

31. J. Adam et al., Eur. Phys. J. C 76(2), 86 (2016). https://doi.org/10. 1140/epjc/s10052-016-3915-1

32. B. Andersson, G. Gustafson, G. Ingelman, T. Sjostrand, Phys. Rep. 97, 31 (1983). https://doi.org/10.1016/0370-1573(83)90080-7

33. T. Sjostrand, S. Mrenna, P.Z. Skands, Comput. Phys. Commun. 178, 852 (2008). https://doi.org/10.1016/j.cpc.2008.01.036

34. T. Sjöstrand, S. Ask, J.R. Christiansen, R. Corke, N. Desai, P. Ilten, S. Mrenna, S. Prestel, C.O. Rasmussen, P.Z. Skands, Comput. Phys. Commun. 191, 159 (2015). https://doi.org/10.1016/j.cpc.2015.01. 024

35. T. Matsui, H. Satz, Phys. Lett. B 178, 416 (1986). https://doi.org/ 10.1016/0370-2693(86)91404-8

36. M.C. Abreu et al., Phys. Lett. B 477, 28 (2000). https://doi.org/10. 1016/S0370-2693(00)00237-9

37. A. Adare et al., Phys. Rev. Lett. 98, 232301 (2007). https://doi.org/ 10.1103/PhysRevLett.98.232301

38. X. Dong, Y.J. Lee, R. Rapp, Ann. Rev. Nucl. Part. Sci. 69, 417 (2019). https://doi.org/10.1146/annurev-nucl-101918-023806

39. R. Vogt, Phys. Rev. C 98(3), 034907 (2018). https://doi.org/10. 1103/PhysRevC.98.034907

40. Y. Akamatsu, T. Hatsuda, T. Hirano, Phys. Rev. C 80, 031901 (2009). https://doi.org/10.1103/PhysRevC.80.031901

41. X. Zhu, M. Bleicher, S.L. Huang, K. Schweda, H. Stoecker, N. Xu, P. Zhuang, Phys. Lett. B 647, 366 (2007). https://doi.org/10.1016/ j.physletb.2007.01.072

42. G. Tsiledakis, H. Appelshauser, K. Schweda, J. Stachel, Nucl. Phys. A 858, 86 (2011). https://doi.org/10.1016/j.nuclphysa.2011. 03.013

43. M. Ruggieri, S.K. Das, EPJ Web Conf. 192, 00017 (2018). https:// doi.org/10.1051/epjconf/201819200017

44. J. Adolfsson et al., Eur. Phys. J. A 56(11), 288 (2020). https://doi. org/10.1140/epja/s10050-020-00270-1

45. J. Adolfsson, Study of $\Xi$-Hadron Correlations in pp Collisions at $\sqrt{s}=13 \mathrm{TeV}$ Using the ALICE Detector. Ph.D. thesis, Lund University (2020). http://cds.cern.ch/record/2750097/

46. J. Adolfsson, Acta Phys. Polon. Supp. 14, 21 (2021). https://doi org/10.5506/APhysPolBSupp.14.21

47. S. Basu, V. Gonzalez, J. Pan, A. Knospe, A. Marin, C. Markert, C. Pruneau, (2020). arXiv:2001.07167 [nucl-ex]

48. T. Sjostrand, S. Mrenna, P.Z. Skands, JHEP 05, 026 (2006). https:// doi.org/10.1088/1126-6708/2006/05/026

49. R. Aaij et al., JHEP 06, 141 (2012). https://doi.org/10.1007/ JHEP06(2012)141. [Addendum: JHEP 03, 108 (2014)]

50. J. Schukraft, A. Timmins, S.A. Voloshin, Phys. Lett. B 719, 394 (2013). https://doi.org/10.1016/j.physletb.2013.01.045

51. B. Abelev et al., Phys. Rev. Lett. 109, 072301 (2012). https://doi. org/10.1103/PhysRevLett.109.072301

52. B.B. Abelev et al., Phys. Lett. B 734, 314 (2014). https://doi.org/ 10.1016/j.physletb.2014.05.064 
53. P. Braun-Munzinger, J. Stachel, Phys. Lett. B 490, 196 (2000). https://doi.org/10.1016/S0370-2693(00)00991-6

54. E. Norrbin, T. Sjostrand, Phys. Lett. B 442, 407 (1998). https://doi. org/10.1016/S0370-2693(98)01244-1
55. S.G. Weber, A. Dubla, A. Andronic, A. Morsch, Eur. Phys. J. C 79(1), 36 (2019). https://doi.org/10.1140/epjc/ s10052-018-6531-4 